\title{
Rock Cutting Analysis Employing Finite and Discrete
}

\section{Element Methods}

Carla Massignani Carrapatoso, Carlos Emmanuel Ribeiro Lautenschläger, Guilherme Lima Righetto, Sergio Augusto Barreto da Fontoura and Nelson Inoue

Civil Engineering Department, Pontifical Catholic University of Rio de Janeiro, Rio de Janeiro 22451-900, Brazil

\begin{abstract}
The petroleum industry has shown great interest in the study of drilling optimization on pre-salt formations given the low rates of penetration observed so far. Rate of penetration is the key to economically drill the pre-salt carbonate rock. This work presents the results of numerical modeling through finite element method and discrete element method for single cutter drilling in carbonate samples. The work is relevant to understand the mechanics of drill bit-rock interaction while drilling deep wells and the results were validated with experimental data raised under simulated downhole conditions. The numerical models were carried out under different geometrical configurations, varying the cutter chamfer size and back-rake angles. The forces generated on the cutter are translated into mechanical specific energy as this parameter is often used to measure drilling efficiency. Results indicate that the chamfer size does not change significantly the mechanical specific energy values, although the cutter aggressiveness is influenced by this geometrical characteristic. Results also show there is a significant increase in drilling resistance for larger values of back-rake angle.
\end{abstract}

Key words: Rock cutting, discrete element method, finite element method, single cutter test.

\section{Introduction}

The drilling optimization depends on a detailed evaluation process of the cutter behavior during the rock cutting. Through single cutter tests, the effects of geometrical variations in the cutter can be evaluated from the point of view of forces and energy. The possibility of assessing these phenomena numerically, through triaxial calibrations of complex models, significantly expands the spectrum of rock drilling optimization process.

The mechanical interaction between the rock sample and the cutter is evaluated in terms of MSE (mechanical specific energy) and aggressiveness. MSE is a parameter commonly used to measure cutting efficiency and can be defined as the work done per unit volume of excavated rock [8]. The aggressiveness, in a single cutter scenario, can be defined as the slope of the plot of cutting force to normal force [1] and,

Corresponding author: Carla Carrapatoso, M.Sc., civil engineer, research fields: discrete element method, rock cutting, and rock mechanics. conventionally, the more aggressive the cutter, the higher the penetration rate achieved. In numerical modeling of single cutter test, keeping constant the depth of cut and the rate of penetration among simulations, the aggressiveness can be used as a sensitivity parameter in analyses regarding the cutting efficiency.

In the present work, numerical triaxial tests were calibrated based on experimental results of Carthage limestone published by Ledgerwood [6]. Model results were also compared to experimental results of single cutter tests found in the literature. Akbari et al. [1] and Akbari et al. [2] performed single cutter tests on Carthage limestone and discussed the influence of back-rake angle and of chamfer size on cutting aggressiveness and on MSE. The aforementioned works corroborated the behavior observed in the results obtained from numerical modeling.

The main motivation of this work is to provide a better understanding on the mechanical behavior of the rock through a numeric study of rock cutting process. Carbonate rocks were chosen to perform this study, 
especially due to its broad presence in the new scenarios of geological exploration, as the pre-salt.

\section{Numerical Calibration}

This item discusses the challenges and the improvements made on the numerical calibration process in order to better represent a synthetic sample of carbonate through the use of discrete element method and finite element method. Triaxial simulations were performed under different confinement and stress-strain curves were compared with the experimental ones.

The triaxial modeling through discrete element method presents some challenges regarding the simulation of the stress-strain inelastic region of a cemented granular material under confinement. The contact models available in PFC (particle flow code) software are not able to capture the post peak flow stress and the clump logic was applied to the synthetic sample to improve the calibration of that region. Clump simulates a group of slave particles joined by their contact points (overlap). They behave as a rigid body, keeping a fixed distance from each other regardless of the forces acting upon them. This technique in conjunction with the linear contact model resulted on a set of micro-properties (Table 1) that represent Carthage limestone numerically. Fig. 1 presents the numerical calibration developed using DEM.

Finite element analyses were performed employing the software ABAQUS. Drucker-Prager yield criterion was used to model the triaxial tests, performed by Ledgerwood [6], by the finite element method. To reproduce accurately experimental tests, a hardening rule was included in ABAQUS models, where test data were entered as tables. The tables were composed by yield stress versus equivalent plastic strain values at different equivalent plastic strain rates.

Damage mechanics concepts were also employed in this calibration. According to Ref. [3], the mechanical behavior of rocks is strongly affected by the presence of heterogeneities such as pores and micro cracks.

These heterogeneities may be responsible for the reduction of the rock strength, when external forces are applied on body. Physically, the damage is directly related to plastic and irreversible strains and is evidenced by reduction of stiffness and material strength. Therefore, the damage initiation criteria adopted in this work was based on Ref. [3] and can be understood as the midpoint between the yield stress and the ultimate stress at the stress-strain curve. In addition, for the synthetic sample of Carthage Marble, the damage evolution was ruled by the elasticity degradation; this parameter determines when the element is removed from the mesh. The final calibration of numerical triaxial test is shown in Fig. 2. The numerical results have a good concordance with the experimental results.

Table 1 Micro properties used to create synthetic rock samples.

\begin{tabular}{lll}
\hline Parameters & Symbol & PFC3D \\
\hline Minimum ball radius (mm) & $R_{\min }$ & 0.6 \\
Ball size ratio & $R_{\max } / R_{\min }$ & 1.3 \\
Ball density $\left(\mathrm{kg} / \mathrm{m}^{3}\right)$ & & 2,680 \\
Ball-ball contact modulus (GPa) & $E_{C}$ & $50 \times 109$ \\
Ball stiffness ratio (GPa) & $K_{n} / K_{s}$ & 3.7 \\
Parallel bond modulus (GPa) & $E_{C}$ & $50 \times 109$ \\
Parallel bond stiffness ratio & $k_{n} / k_{s}$ & 3.7 \\
Ball friction coefficient & & 1 \\
Parallel bond normal strength, mean (MPa) & $\sigma_{c, \text { mean }}$ & $130 \times 106$ \\
Parallel bond normal strength, std. dev. (MPa) & $\sigma_{c, \text { std.dev. }}$ & $130 \times 106$ \\
Parallel bond shear strength, mean (MPa) & $\tau_{c, \text { mean }}$ & $60 \times 106$ \\
Parallel bond shear strength, std. dev. (MPa) & $\tau_{c, \text { std.dev. }}$ & $60 \times 106$ \\
\hline
\end{tabular}




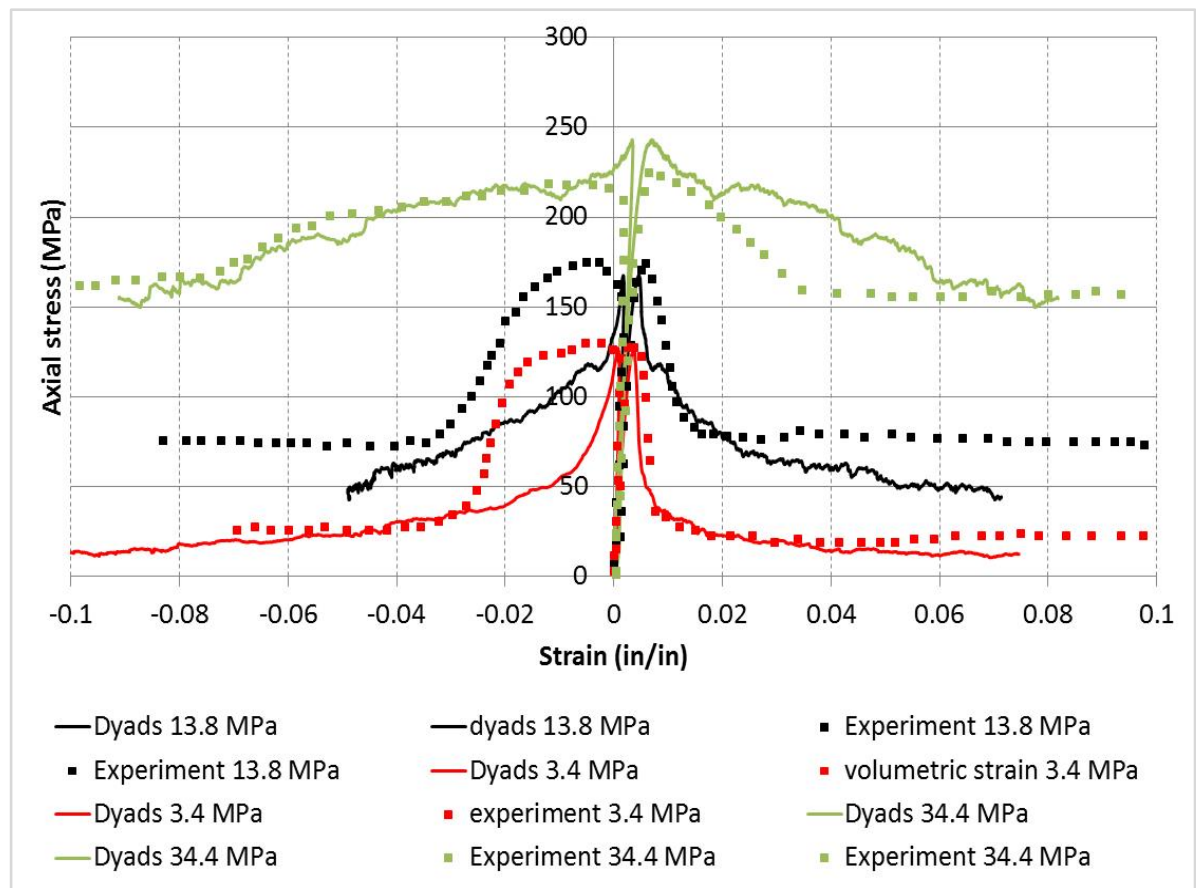

Fig. 1 Triaxial test modeling using discrete element method.

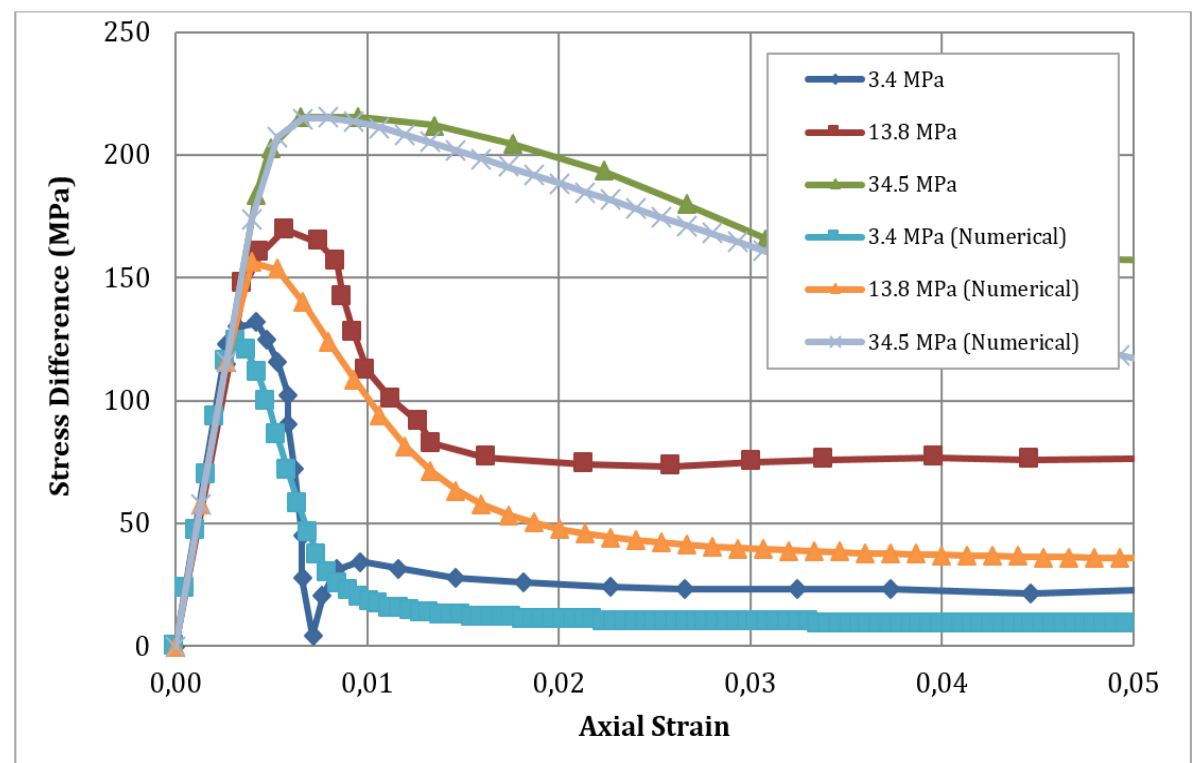

Fig. 2 Triaxial test modeling using discrete element method.

It is important to highlight that on a FEM single cutter simulation, the cutter moves in vertical, horizontal, and angular directions, thus it is not possible to define a fixed cutting surface. Therefore, an erosion contact model was adopted to overcome this difficulty. In summary, the numerical process of rock cutting can be divided in three main stages: the element failure, element removal, and the contact surface updating on the exterior face of elements that have not failed.

\section{Model Geometry}

All effects evaluated in this paper are strongly dependent of the geometrical arrangement of the rock-cutter set. Fig. 3 illustrates two perspectives of the general geometry adopted for all models simulated in 


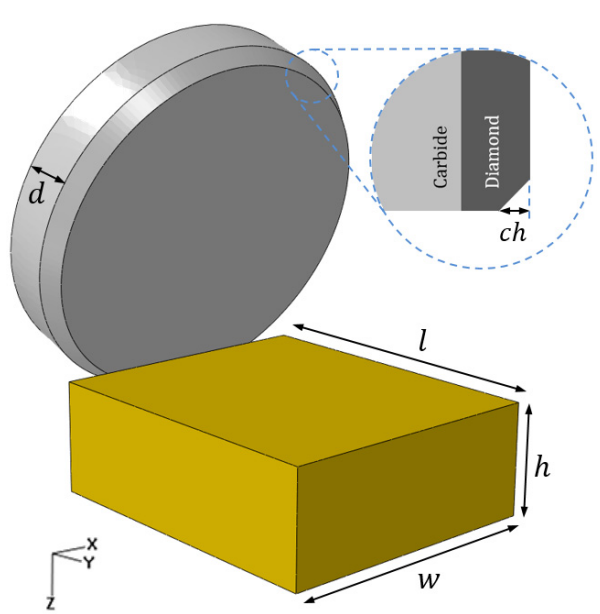

(a) Front view

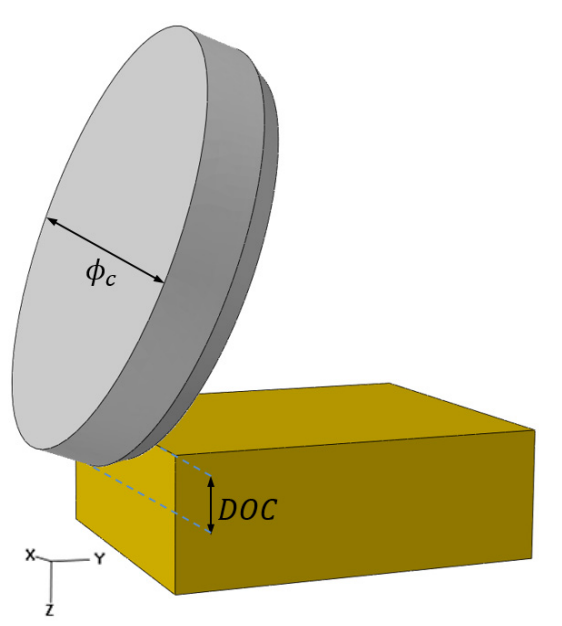

(b) Back view

Fig. 3 Geometric characteristics of the model.

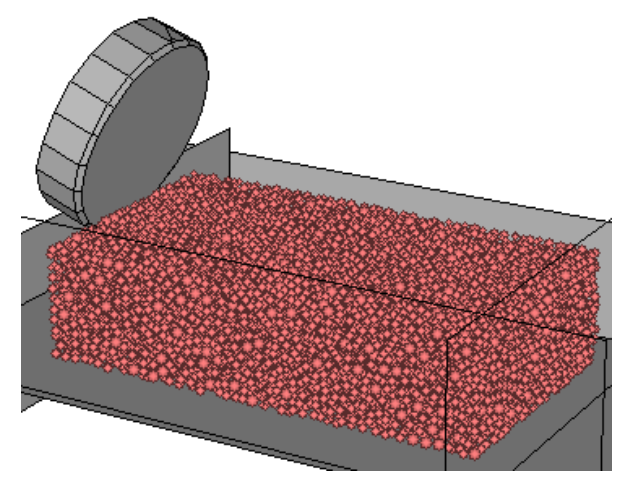

(a) DEM assembly

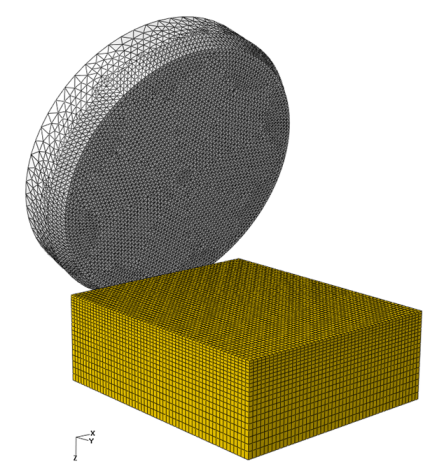

(b) FEM mesh

Fig. 4 Numerical models.

this work. For each model, the variables depicted in the sketch assume different values. The mentioned variables are:

- $c h$-length of the chamfer of PDC cutter;

- $d$-thickness of the cutter in the full section portion;

- $l$-length of the rock sample;

- $h$-height of the rock sample;

- $w$-width of the rock ample;

- $\phi_{c}$-cutter diameter;

- DOC - depth of cut from the lowest chamfer edge.

Different models were built to perform a parametric analysis, with the cutter geometry and orientation varying in each simulation. The DOC and the confining pressure were kept constant in the numerical analyses ( $0.05 \mathrm{in}$ and $3000 \mathrm{psi}$, respectively). The dimensions $l, h$ and $w$ are, respectively, 35, 15 and $30 \mathrm{~mm}$ to DEM analyses, and 14, 4.8 and $12 \mathrm{~mm}$ to FEM analyses.
Variables $d$ and $\phi_{c}$ are equivalent to $3 \mathrm{~mm}$ and $16 \mathrm{~mm}$, respectively in both numerical methods.

To assess the chamfer effect, this variable was changed among models, assuming values of $0.010 \mathrm{in}$, $0.016 \mathrm{in}$ and 0.019in. A sharp cutter was also used in this comparison. The back-rake angles (the angle between the vertical direction and the cutter face) evaluated were $10^{\circ}, 20^{\circ}$ and $30^{\circ}$. The aggressiveness was evaluated in conjunction with chamfer size and back-rake variations, as will be explained in the results section. All aforementioned variations were applied in both methods. Fig. 4a shows an example of a discrete element model and Fig. 4b presents an example of finite element model as well.

The dimensions adopted in each numerical model are consistent with the refinement required for each method. In the discrete element models, the same 
synthetic sample was used for all simulations in order to minimize uncertainties related to the particles positioning. In the finite element analysis, the cutter and rock sample refinement were optimized by using a larger refinement in the chamfer and groove region, improving numerical processes.

\section{Results}

In this section, the influence of cutter geometry on cutting efficiency will be discussed. The effects of chamfer and back-rake angle were evaluated through discrete and finite element modeling, using the geometrical parameters and the model configuration presented in the former section. The variables effect was evaluated through MSE and aggressiveness.

\subsection{Influence of Chamfer Geometry}

Figs. 5 and 6 present the evolution of MSE with chamfer size for the discrete and finite element method, respectively. It is important to emphasize that all other modeling parameters were kept constant in order to capture the effect of chamfer size on cutting efficiency. Results show that the use of chamfer did not present influence over the MSE for the evaluated range of values, once the graphics trend is a plateau. The MSE remained constant around $600 \mathrm{MPa}$ for FEM results and around 1,050 MPa for DEM evaluated cases.

The difference of magnitudes between FEM and DEM modeling values is probably related to the great quantity of accumulated particles stuck to the cutter's face during DEM simulations. These materials generate extra forces increasing MSE values. A comparison between MSE values found on modeling and on experimental test should be done in order to calibrate the amount of accumulated particles during simulation.

Figs. 7 and 8 show plots of vertical force versus horizontal forces, wherein the slope of the trend line consists of the cutter aggressiveness. The consideration of the chamfer resulted in a slight effect over the cutter aggressiveness. According to Ref. [1], the cutter aggressiveness increases as the size of the chamfer decreases. Observing the FEM and DEM plotted curves the more inclined curves indicate more aggressive cutter behavior. In the FEM analyses, the cutter with chamfer size of 0.010in was more aggressive than the cutter with chamfer of 0.016in, as reported by Akbari et al. [1]. The behavior presented by the cutter with chamfer of 0.019in appears to be inconsistent when compared to another two chamfer sizes studied, possibly due to the elevated contribution of this chamfer in the cutting area.

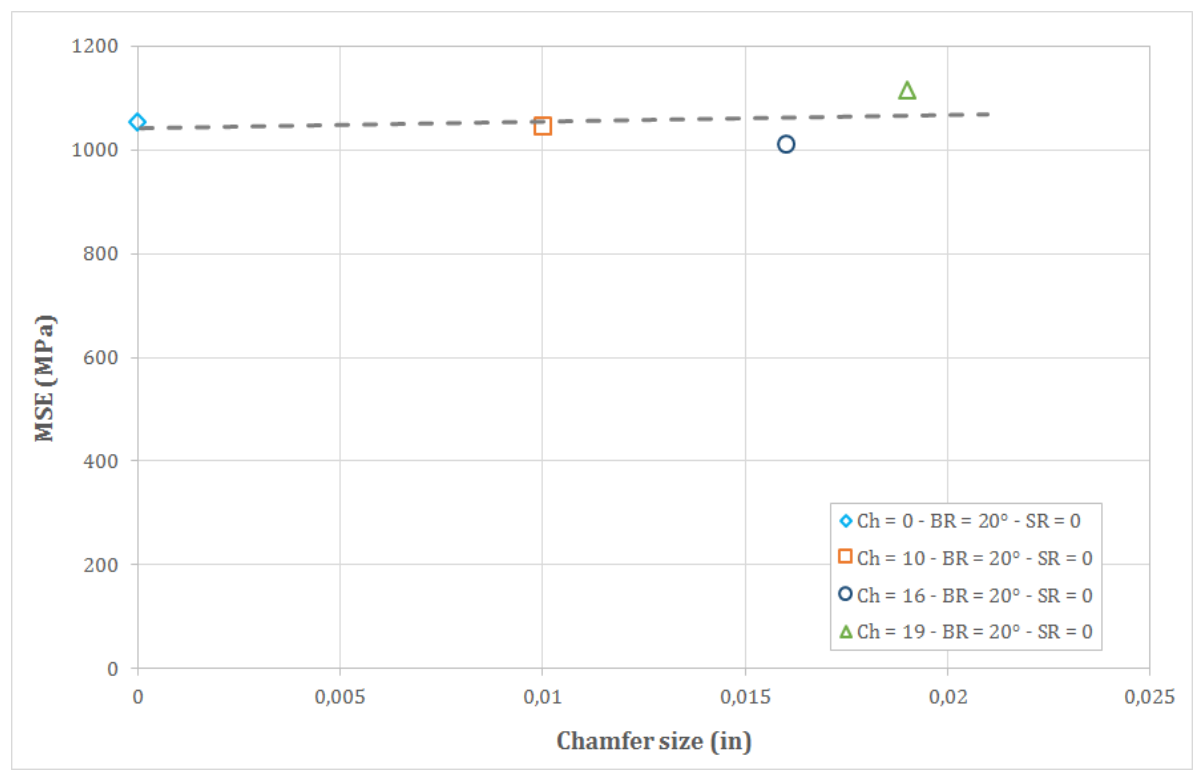

Fig. 5 Influence of chamfer size on mechanical specific energy using discrete element analysis. 


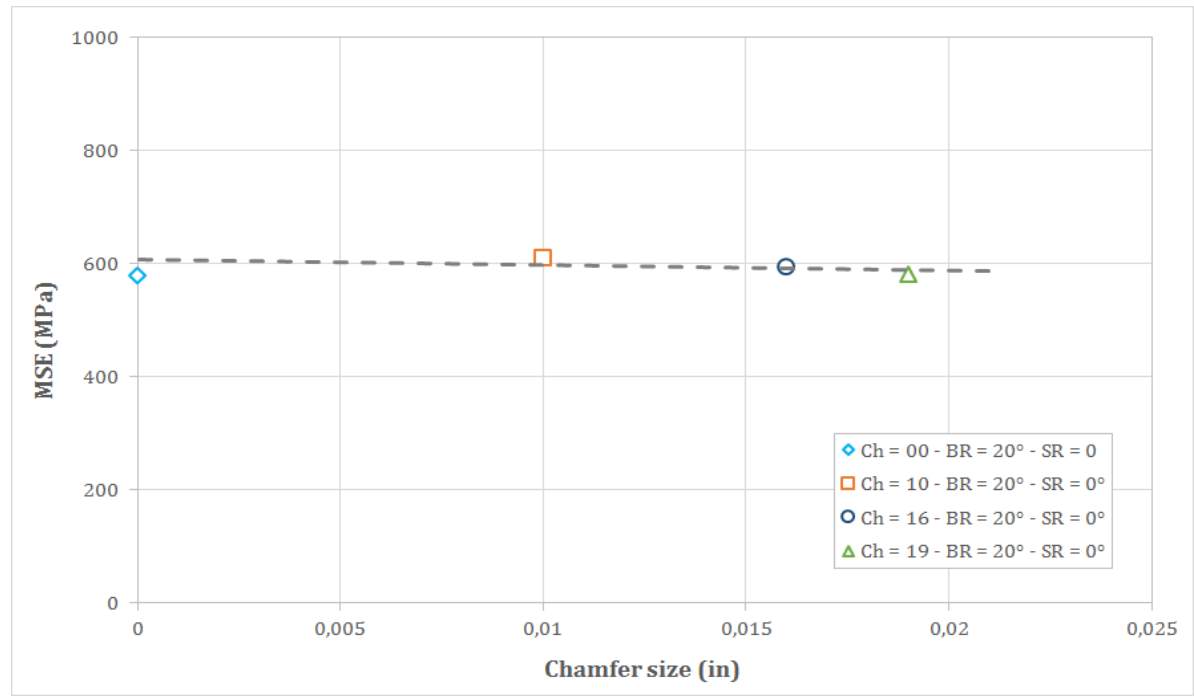

Fig. 6 Influence of chamfer size on mechanical specific energy using finite element analysis.

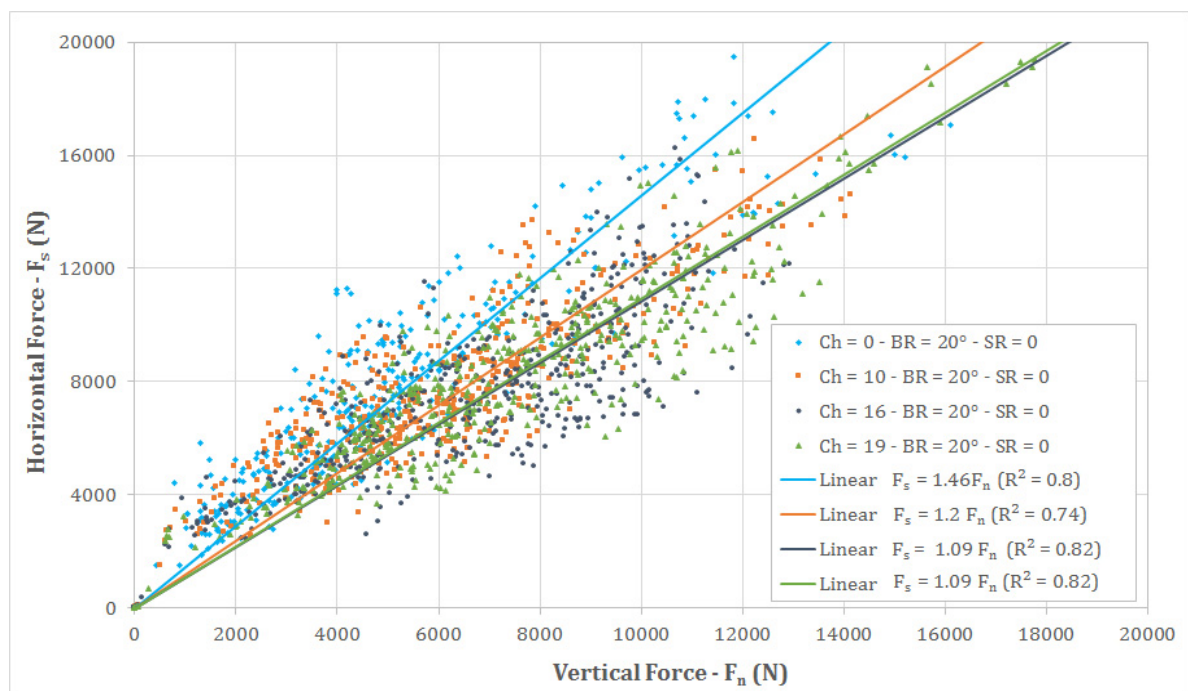

Fig. 7 Influence of the chamfer size on the cutter aggressiveness using discrete element analysis.

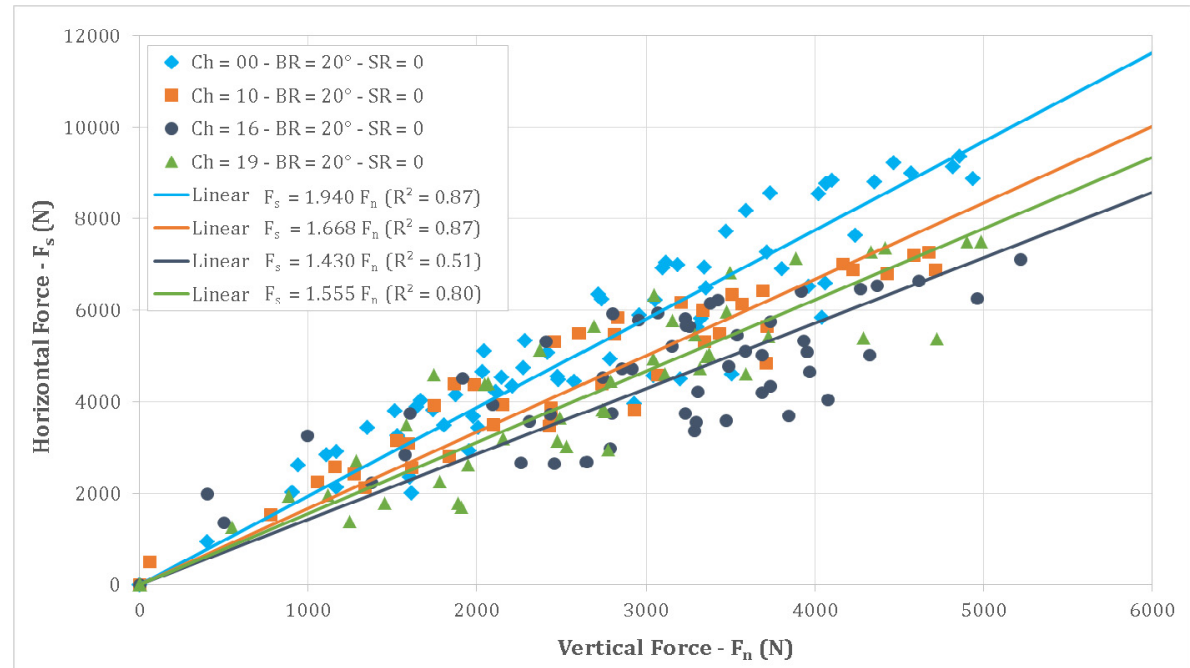

Fig. 8 Influence of the chamfer size on the cutter aggressiveness using finite element analysis. 


\subsection{Influence of Back-Rake Angle}

Figs. 9 and 10 present the effect of back-rake angle on MSE generation. MSE increases with the cutter inclination, as expected. This type of analysis was previously carried out by other authors in different rock types $[4,5,7]$ and similar trends were found.

Figs. 11 and 12 show the influence of back-rake angle on the cutter aggressiveness, obtained by plotting horizontal forces versus vertical forces for both DEM and FEM. It was observed that back-rake variation did not present effects over the cutter aggressiveness, once the slope of the adjustment lines presented, practically, the same value (aggressiveness angle around $55^{\circ}$ and $47^{\circ}$ for FEM and DEM analyses, respectively).

Literature results corroborate this behavior. According to Ref. [5], the relation between horizontal and vertical forces does not vary with back-rake angle: the angle of resultant force relative to the displacement direction remains the same regardless back-rake angle.

Analyzing Fig. 13 which illustrates the force vectors in the horizontal, vertical and resultant directions on the cutter, it is evident the conclusion performed by

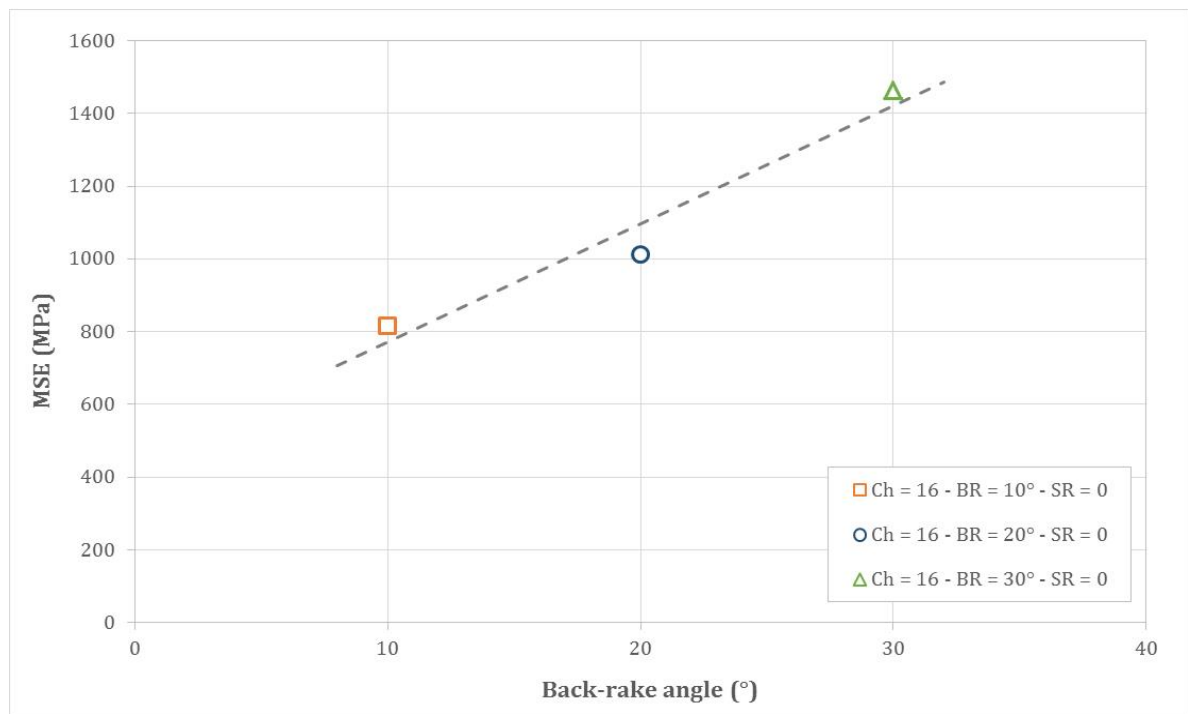

Fig. 9 Influence of back-rake angle on mechanical specific energy using discrete element analysis.

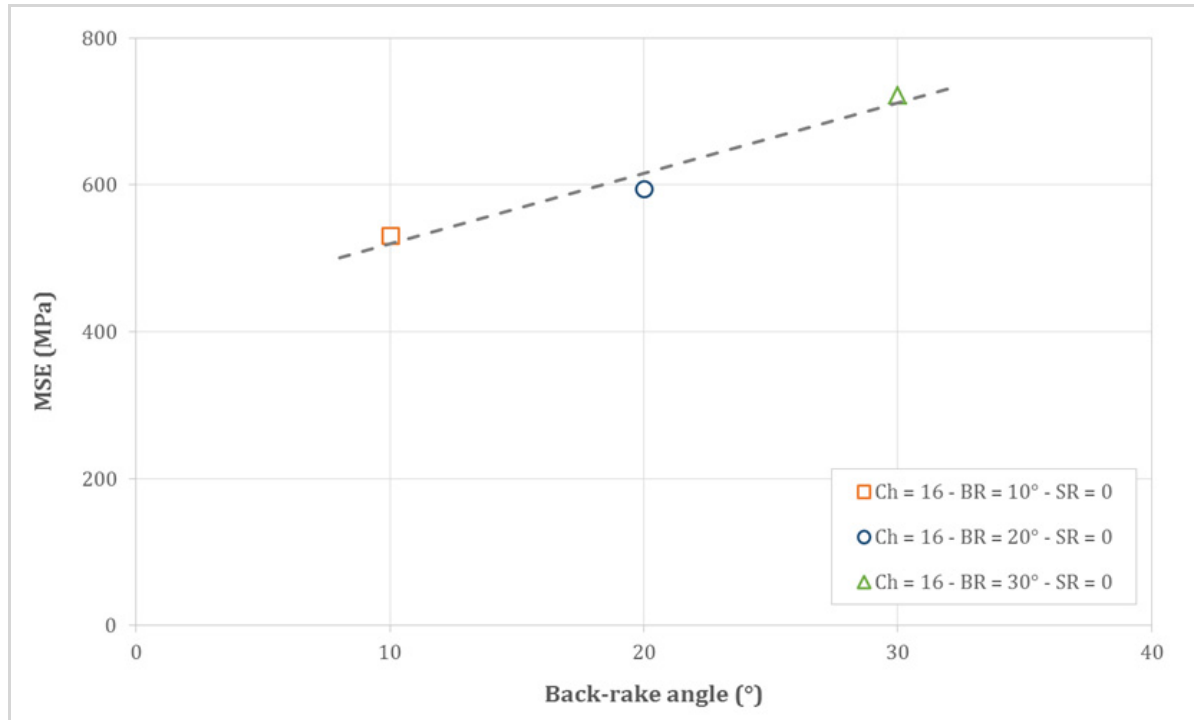

Fig. 10 Influence of back-rake angle on mechanical specific energy using finite element analysis. 


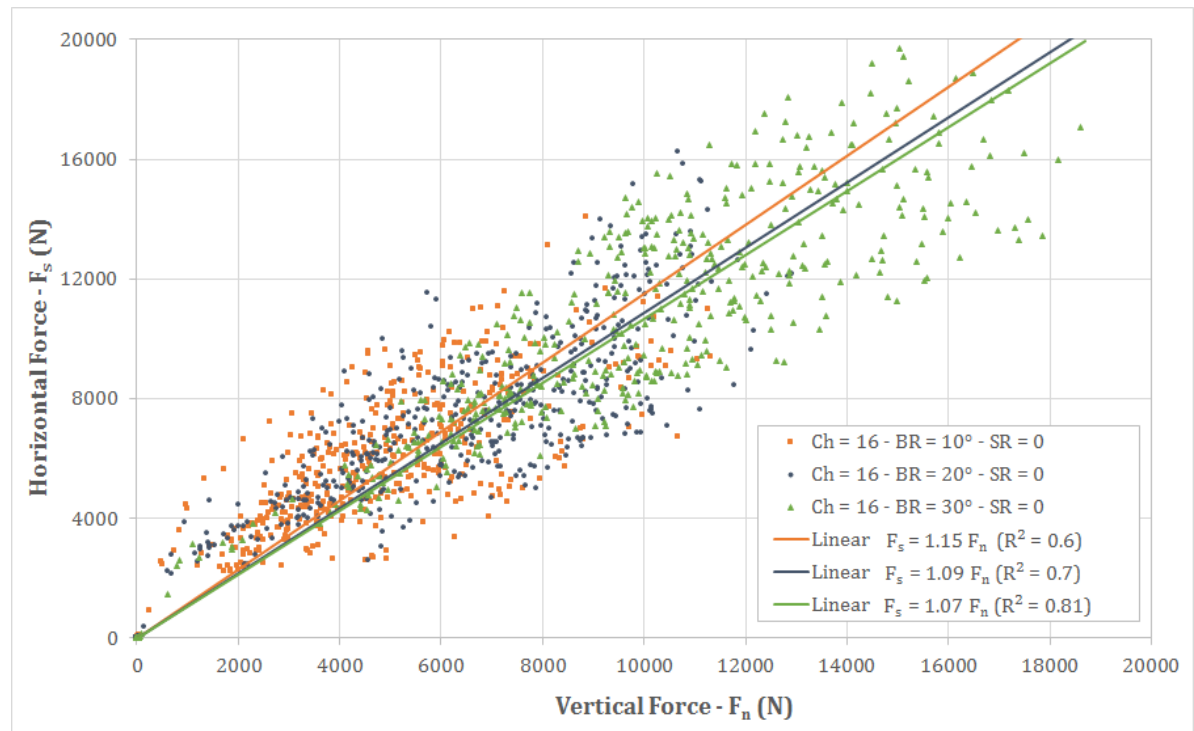

Fig. 11 Influence of the back-rake angle on the cutter aggressiveness using discrete element analysis.

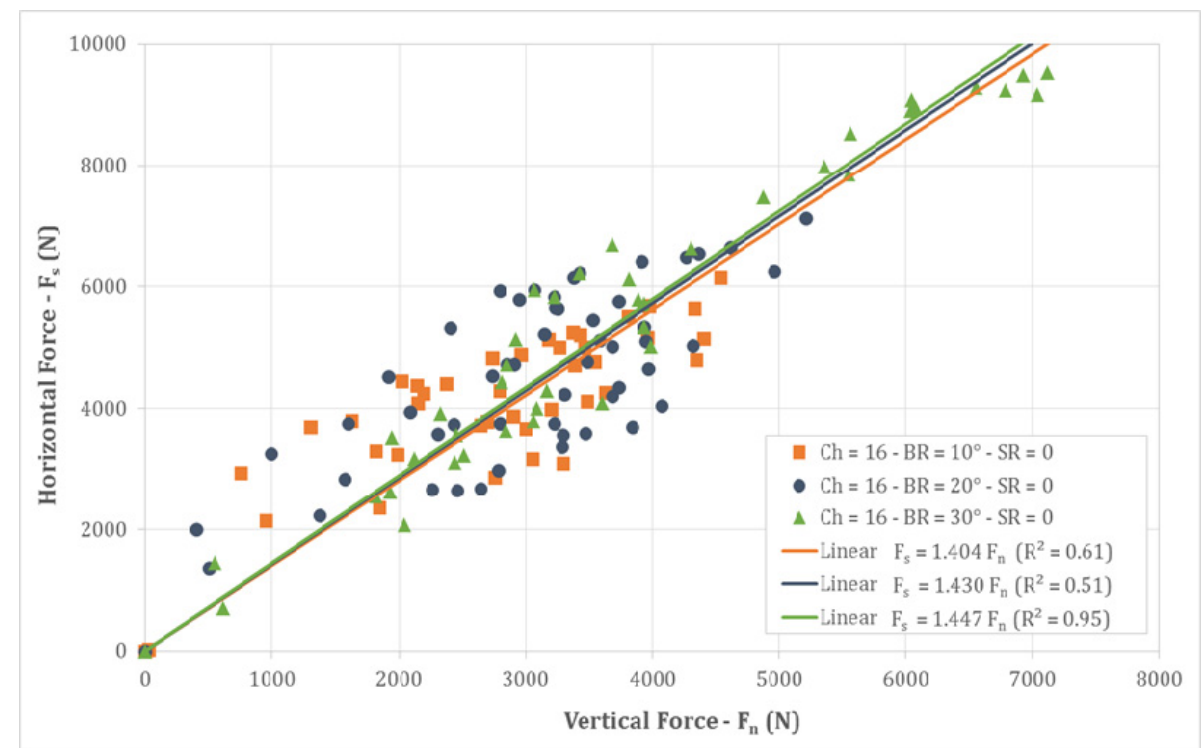

Fig. 12 Influence of the back-rake angle on the cutter aggressiveness using finite element analysis.

Ghoshouni et al. [5] regarding the maintenance of the resultant force orientation despite the back-rake variation.

\section{Conclusions}

In general, the modeling using DEM and FEM presented good agreement with literature results, especially regarding the trends obtained in the parametrical analysis. The absolute values of MSE are high when compared to those obtained by FEM for large confinement and single cutter experiments are required to compare magnitudes.
The cutter aggressiveness was evaluated through the ratio between tangential and vertical force components. Results showed that the decrease of chamfer size is related to higher aggressiveness, which indicates that higher penetration rates can be achieved for this condition. The aggressiveness was not sensitive to back-rake angle changing. It is important to remember that modeling does not consider an application of vertical velocity in the cutter, as it happens experimentally. In this case, results can be different and should be modeled. 


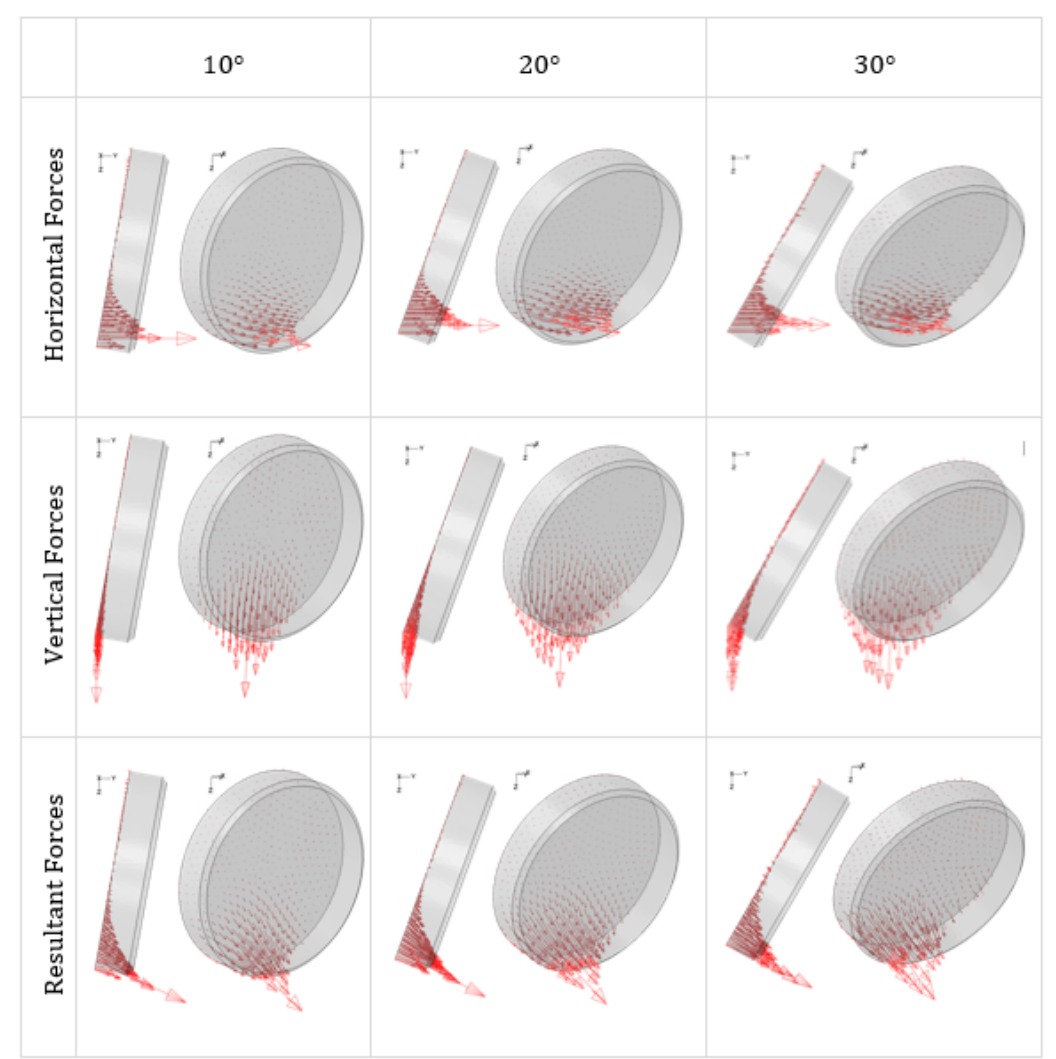

Fig. 13 Influence of the back-rake angle on the resultant force direction using finite element analysis.

The effect of different chamfer sizes was evaluated aiming to understand force components and MSE contributions to the cutting efficiency. Results show that MSE is slightly altered by chamfer size changing, indicating that a cutter with a chamfer does not make rock cutting less efficient in the evaluated scenarios.

\section{References}

[1] Akbari, B., Miska, S. Z., Yu, M., and Rahmani, R. 2014. "The Effects of Size, Chamfer Geometry, and Back-rake Angle on Frictional Response of PDC Cutters." ARMA 14-7458, Presented at the 48th US Rock Mechanics/Geomechanics Symposium, Minneapolis, MN.

[2] Akbari, B., Miska, S. Z., Yu, M., and Ozbayoglu, E. 2014. "Relation between the Mechanical Specific Energy, Cuttings Morphology and Cutter Geometry." OMAE 14-24708, Presented at the 33rd International Conference on Ocean, Offshore and Artic Engineering, San Francisco, CA.

[3] Aubertin, M., and Simon, R. 1997. "A Damage Initiation Criterion for Low Porosity Rock." International Journal of Rock Mechanics and Mining Sciences 34: 3-4.
[4] Carrapatoso, C. M., Fontoura, S., Inoue, N., Martinez, I. M. R., Lourenço, A., and Curry, D. 2013. "Simulation of Single Cutter Experiments in Evaporite using the Discrete Element Method." Presented at the EUROCK 2013-The 2013 ISRM International Symposium-Rock Mechanics for Resources, Energy, and Environment.

[5] Ghoshouni, M., and Richard, T. 2008. "Effect of Back Rake Angle and Groove Geometry in Rock Cutting." Presented at the ISRM International Symposium, Iran.

[6] Ledgerwood III, L. W. R. 2013. "PFC3D Model of Rock Cutting under Pressure Calibrated to the Inelastic Region of Triaxial Tests at High Strain." Presented at 6th International Conference on Discrete Element Methods, August 6.

[7] Rajabov, V., Miska, S., Mortimer, L., Yu, M., and Ozbayoglu, E. 2012. "The Effects of Back Rake and Side Rake Angles on Mechanical Specific Energy of Single PDC Cutters with Selected Rocks at Varying Depth of Cuts and Confining Pressures." IADC/SPE 151406, Presented at the 2012 IADC/SPE Drilling Conference and Exhibition, USA.

[8] Teale, R. 1965. "The Concept of Specific Energy in Rock Drilling." International Journal of Rock Mechanics and Mining Science 2: 57-73. 